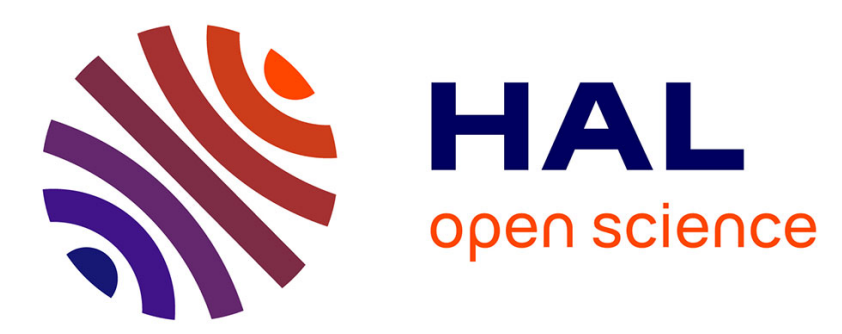

\title{
Analyse de la structure rotationnelle de la bande de vibration-rotation v3 du dioxyde d'azote
}

V. Dana

\section{To cite this version:}

V. Dana. Analyse de la structure rotationnelle de la bande de vibration-rotation v3 du dioxyde d'azote. Revue de Physique Appliquée, 1974, 9 (4), pp.711-720. 10.1051/rphysap:0197400904071100 . jpa00243838

\section{HAL Id: jpa-00243838 https://hal.science/jpa-00243838}

Submitted on 1 Jan 1974

HAL is a multi-disciplinary open access archive for the deposit and dissemination of scientific research documents, whether they are published or not. The documents may come from teaching and research institutions in France or abroad, or from public or private research centers.
L'archive ouverte pluridisciplinaire HAL, est destinée au dépôt et à la diffusion de documents scientifiques de niveau recherche, publiés ou non, émanant des établissements d'enseignement et de recherche français ou étrangers, des laboratoires publics ou privés. 


\title{
ANALYSE DE LA STRUCTURE ROTATIONNELLE DE LA BANDE DE VIBRATION-ROTATION $v_{3}$ DU DIOXYDE D'AZOTE
}

\author{
V. DANA \\ Laboratoire de Spectroscopie Moléculaire \\ Université de Paris VI, 4, place Jussieu, 75230 Paris Cedex 05, France
}

(Reçu le 2 janvier 1974, révisé le 29 janvier 1974)

\begin{abstract}
Résumé. - Le travail présenté ici est une analyse de la structure rotationnelle de la bande de vibration $v_{3}$ du dioxyde d'azote centrée vers $1616,844 \mathrm{~cm}^{-1}$. Le spectre d'absorption de cette bande, présenté dans une précédente publication [1], a été enregistré à l'aide d'un spectromètre à grilles dont la largeur de la fonction d'appareil est d'environ $0,06 \mathrm{~cm}^{-1}$.

L'ajustement des constantes relatives au niveau supérieur de la transition a été réalisé à l'aide d'un programme de moindres carrés itératif dans lequel le calcul des énergies des niveaux rotationnels est fondé sur le formalisme de Watson [2].

Les nombres d'ondes calculés à partir de ces constantes sont en très bon accord avec les résultats expérimentaux.
\end{abstract}

\begin{abstract}
The spectroscopic constants of the (001) level of nitrogen dioxide are computed from a high resolution absorption spectrum of the $v_{3}$ band around $1616 \mathrm{~cm}^{-1}$ recorded on a dualgrill spectrometer. The spectrum (described previously [1]) was obtained with a resolution of $0.06 \mathrm{~cm}^{-1}$. The constants are computed by a least squares method. These constants enabled us to obtain theoretical wave numbers which are in good agreement with the experimental results.
\end{abstract}

1. Introduction. - L'analyse de la structure rotationnelle du spectre d'absorption du dioxyde d'azote a fait l'objet de nombreux travaux. Parmi les plus récents citons ceux effectués par Bird et coll. [3] sur le spectre de rotation pure et ceux de Blank, Olman et Hause [4-5-6] dans l'infrarouge proche.

La bande de vibration-rotation $v_{3}$ a, d'autre part, été étudiée par E. T. Arakawa et A. H. Nielsen [7] et plus récemment par Hurlock [8]. Cabana [9] s'est intéressé à la structure rotationnelle de la bande $v_{2}$ et a réalisé une synthèse des travaux relatifs à l'état vibrationnel fondamental, il a obtenu les constantes présentées dans le tableau I. Dans le cadre de l'étude spectroscopique des constituants minoritaires de l'atmosphère, réalisée en collaboration avec l'Office

\section{TABLEAU I}

Constantes relatives à l'état vibrationnel (000), proposées par Cabana $\left(e n \mathrm{~cm}^{-1}\right)$.

$\begin{array}{ll}\mathrm{A} & 8,00237 \\ \mathrm{~B} & 0,4337059 \\ \mathrm{C} & 0,41004485 \\ \Delta_{K} & 2,6901 \times 10^{-3} \\ \Delta_{N K} & -1,9579 \times 10^{-5} \\ \Delta_{N} & 3,112 \times 10^{-7} \\ \delta_{K} & 2,4 \times 10^{-8} \\ \delta_{N} & 3,022 \times 10^{-8} \\ H_{K} & 2,157 \times 10^{-6}\end{array}$

National d'Etudes et de Recherches Aérospatiales, nous avons entrepris l'analyse la plus complète possible de la structure rotationnelle de la bande $v_{3}$ et déterminé les valeurs des constantes relatives au niveau vibrationnel 001 .

2. Description du spectre d'absorption. - La molécule $\mathrm{NO}_{2}$ est une toupie légèrement asymétrique ( $\kappa \simeq-0,994)$ plane, appartenant au groupe de symétrie $\mathrm{C}_{2 \mathrm{v}}$. Cette molécule possède un électron libre, ce qui entraîne un dédoublement des niveaux rotationnels et par conséquent un dédoublement des raies de vibration-rotation.

La bande étudiée est du type $\mathrm{A}$; les règles de sélection qui régissent les transitions vibro-rotationnelles sont, avec une excellente approximation, celles des bandes parallèles des toupies symétriques

$$
\begin{array}{llll}
\Delta N & =0 \pm 1 & \text { si } & K_{-1} \neq 0 \\
\Delta N & = \pm 1 & \text { si } & K_{-1}=0 \\
\Delta K_{-1} & =0 & &
\end{array}
$$

$N$ caractérise le moment angulaire de rotation pure contrairement à $J$ qui tient compte de la rotation de la molécule et du moment angulaire de spin électronique.

Rappelons que dans le cas de $\mathrm{NO}_{2}$, dans un état vibrationnel donné, un niveau sur deux est peuplé. Ces niveaux sont définis par les relations suivantes:

$$
\begin{aligned}
& K_{-1}+K_{+1}=\text { pair pour l'état vibrationnel } 000 \\
& K_{-1}+K_{+1}=\text { impair pour l'état vibrationnel } 001
\end{aligned}
$$


Le spectre d'absorption de la bande $v_{3}$, a été enregistré à l'ONERA par J. C. Fontanella à l'aide d'un spectromètre à grilles dans l'intervalle spectral compris entre 1587,6 et $1639 \mathrm{~cm}^{-1}$. Ce spectre ainsi qu'une analyse sommaire de la structure rotationnelle ont été présentés dans une précédente publication [1].

Rappelons brièvement les conditions d'enregistrement :

Largeur de la fonction d'appareil : $0,06 \mathrm{~cm}^{-1}$. Rapport signal sur bruit : 50 .

Pression de gaz: quelques millimètres de mercure.

Longueur de cuve : $10 \mathrm{~cm}$.

Température du gaz : $296 \mathrm{~K}$.

Notons que la résolution du spectromètre ne permet pas d'observer le dédoublement des raies de vibrationrotation.

Rappelons que $\mathrm{N}_{2} \mathrm{O}_{4}$, qui est en équilibre chimique avec $\mathrm{NO}_{2}$, est inactif dans la région de $6,18 \mu$. Les bandes de vibration les plus proches étant centrées vers 1260 et $1750 \mathrm{~cm}^{-1}$.

3. Méthode de calcul. Résultats. - La bande $v_{3}$ possède une structure rotationnelle très serrée qui s'étend, si l'on considère les raies les plus intenses $\left(N^{\prime \prime} \leqslant 30\right)$, sur un domaine spectral large d'environ $60 \mathrm{~cm}^{-1}$; il en résulte que les raies isolées utilisables dans nos calculs sont peu nombreuses (160). Cela nous a conduit à limiter notre étude uniquement à la détermination des constantes relatives à l'état vibrationnel (001) en utilisant pour l'état vibrationnel fondamental les constantes proposées par Cabana.

L'ajustement de ces constantes a été réalisé à l'aide d'un programme de moindres carrés itératif établi par C. Camy-Perret et J. M. Flaud, dans lequel le calcul des énergies des niveaux rotationnels est fondé sur le formalisme développé par Watson [2].

Les calculs ont été réalisés dans la représentation $I^{\mathrm{r}}$ la plus adaptée au cas de $\mathrm{NO}_{2}$, l'hamiltonien d'interaction entre le spin électronique et le moment angulaire de rotation n'a pas été introduit dans notre programme. Les poids statistiques des 160 raies isolées prises en considération dans les calculs, sont tous égaux à 1 .

Dans le tableau II figurent les valeurs des constantes relatives à l'état vibrationnel (001) ainsi que leur intervalle de confiance pour un seuil de $99 \%$.

Dans ce tableau n'apparaissent pas les valeurs de $\delta_{K}, \delta_{N}$ et de $H_{K}$, la précision des données expérimentales $\left(0,01 \mathrm{~cm}^{-1}\right.$ environ) ne permettant pas une

\section{TABLEAU II}

Constantes relatives à l'état vibrationnel (001). (Les intervalles de confiance sont donnés pour un seuil de $99 \%$ ) (en $\left.\mathrm{cm}^{-1}\right)$.

$\begin{array}{lll}v_{0} & 1616,8438 & \pm 5,0 \times 10^{-3} \\ \mathrm{~A} & 7,77411 & \pm 4,5 \times 10^{-4} \\ \mathrm{~B} & 0,430945 & \pm 3,2 \times 10^{-5} \\ \mathrm{C} & 0,407883 & \pm 3,4 \times 10^{-5} \\ \Delta_{K} & 2,5634 \times 10^{-3} & \pm 6,9 \times 10^{-6} \\ \Delta_{N K} & -2,523 \times 10^{-5} & \pm 6,8 \times 10^{-7} \\ \Delta_{N} & 3,21 \times 10^{-7} & \pm 3,3 \times 10^{-8}\end{array}$

détermination correcte de ces constantes; nous leur avons attribué les valeurs des constantes correspondantes relatives à l'état vibrationnel fondamental.

L'ensemble des constantes ainsi défini nous a permis de recalculer les nombres d'ondes des transitions qui apparaissent dans l'intervalle spectral enregistré en nous limitant à $N^{\prime \prime} \leqslant 30$ et $R_{-1} \leqslant 8$.

Les nombres d'ondes calculés et observés des raies des branches $P$ et $R$ sont regroupés dans le tableau III. On peut constater dans le cas des raies isolées un bon accord entre les deux ensembles de valeurs, compte tenu des erreurs expérimentales; l'écart quadratique moyen, pour les nombres d'ondes des raies isolées, valant $0,0075 \mathrm{~cm}^{-1}$.

Dans la dernière colonne du tableau III l'astérisque indique que la raie fait partie d'un ensemble de raies non résolu.

Nous avons dénombré une vingtaine de raies des branches $\mathrm{Q}$ isolées que nous avons introduites dans nos calculs; nous ne les avons pas présentées dans le tableau III car elles sont peu intenses et ne présentent pas d'intérêt pour les études spectroscopiques de l'atmosphère.

4. Conclusion. - Les constantes de l'état vibrationnel (001) ont été déterminées avec une précision suffisante pour analyser les spectres atmosphériques dont nous disposons actuellement. Toutefois l'étude d'un spectre mieux résolu de la bande de vibration $v_{3}$ permettra d'améliorer la précision de ces résultats.

Remerciements. - Je remercie J. C. Fontanella de l'O. N.E. R. A. pour sa collaboration sur le plan expérimental, C. Camy-Perret et J. M. Flaud pour les conseils fructueux qu'ils m'ont prodigués, N. Gasgnier des Tables Internationales de Constantes, pour sa contribution technique.

\section{Bibliographie}

[1] Dana, V., Fontanella, J. C., Nouv. Revue Opt. 4 (1973) 237.

[2] Watson, J. K. G., J. Chem. Phys. 46 (1967) 1935.

[3] Bird, G. R., Hunt, G. R., Gebbie, H. A. et Stone, N. W. B., J. Mol. Spectrosc. 33 (1970) 244.

[4] Olman, M. D. et Hause, C. D., J. Mol. Spectrosc. 26 (1968) 241.

[5] Blank, R. E., Olman, M. D. et Hause, C. D., J. Mol. Spectrosc. 33 (1970) 109.
[6] Blank, R. E., Hause, C. D., J. Mol. Spectrosc. 34 (1970) 478.

[7] Arakawa, E. T. et Nielsen, A. H., J. Mol. Spectrosc. 2 (1958) 413.

[8] Hurlock, S. C., Thèse, Ohio State University, Colombus, 1970.

[9] Laurin, M., Cabana, A. et Lafferty, W. J., communication, Colloque de Spectroscopie moléculaire à haute résolution (Tours, sept. 1973). 
TABleAU III $a$.

NOMBRES QUANTIQUES

$\begin{array}{rrrrrr}\mathbf{N} & \mathbf{K}_{-1}^{\prime \prime} & \mathbf{K}_{\mathbf{+ 1}}^{\prime \prime} & \mathbf{N}^{\prime} & \mathbf{K}_{-1}^{\prime} & \mathbf{K}_{+1}^{\prime} \\ 2 & 0 & 2 & 1 & 0 & 1 \\ 2 & 1 & 1 & 1 & 1 & 0 \\ 3 & 1 & 3 & 2 & 1 & 2 \\ 3 & 2 & 2 & 2 & 2 & 1 \\ 4 & 0 & 4 & 3 & 0 & 3 \\ 4 & 1 & 3 & 3 & 1 & 2 \\ 4 & 2 & 2 & 3 & 2 & 1 \\ 4 & 3 & 1 & 3 & 3 & 0 \\ 5 & 1 & 5 & 4 & 1 & 4 \\ 5 & 2 & 4 & 4 & 2 & 3 \\ 5 & 3 & 3 & 4 & 3 & 2 \\ 5 & 4 & 2 & 4 & 4 & 1 \\ 6 & 0 & 6 & 5 & 0 & 5 \\ 6 & 1 & 5 & 5 & 1 & 4 \\ 6 & 2 & 4 & 5 & 2 & 3 \\ 6 & 3 & 3 & 5 & 3 & 2 \\ 6 & 4 & 2 & 5 & 4 & 1 \\ 6 & 5 & 1 & 5 & 5 & 0 \\ 7 & 1 & 7 & 6 & 1 & 6 \\ 7 & 2 & 6 & 6 & 2 & 5 \\ 7 & 3 & 5 & 6 & 3 & 4 \\ 7 & 4 & 4 & 6 & 4 & 3 \\ 7 & 5 & 3 & 6 & 5 & 2 \\ 7 & 6 & 2 & 6 & 6 & 1 \\ 8 & 0 & 8 & 7 & 0 & 7 \\ 8 & 1 & 7 & 7 & 1 & 6 \\ 8 & 2 & 6 & 7 & 2 & 5 \\ 8 & 3 & 5 & 7 & 3 & 4 \\ 8 & 4 & 4 & 7 & 4 & 3 \\ 8 & 5 & 3 & 7 & 5 & 2 \\ 8 & 6 & 2 & 7 & 6 & 1 \\ 8 & 7 & 1 & 7 & 7 & 0 \\ 9 & 1 & 9 & 8 & 1 & 8 \\ 9 & 2 & 8 & 8 & 2 & 7 \\ 9 & 3 & 7 & 8 & 3 & 6 \\ 9 & 4 & 6 & 8 & 4 & 5 \\ 9 & 5 & 5 & 8 & 5 & 4 \\ 9 & 6 & 4 & 8 & 6 & 3 \\ 9 & 7 & 3 & 8 & 7 & 2 \\ 9 & 8 & 2 & 8 & 8 & 1 \\ 10 & 0 & 10 & 9 & 0 & 9 \\ 10 & 1 & 9 & 9 & 1 & 8 \\ 10 & 2 & 8 & 9 & 2 & 7 \\ 10 & 3 & 7 & 9 & 3 & 6 \\ 10 & 4 & 6 & 9 & 4 & 5 \\ 10 & 5 & 5 & 9 & 5 & 4 \\ 10 & 6 & 4 & 9 & 6 & 3 \\ 10 & 7 & 3 & 9 & 7 & 2 \\ 10 & 8 & 2 & 9 & 8 & 1 \\ 11 & 1 & 11 & 10 & 1 & 10 \\ 11 & 2 & 10 & 10 & 2 & 9\end{array}$

NOMBRE DIONDES CALCULE

\subsection{1}

1614.901

1614.103

1613.391

1613.436

1613.163

1612.534

1611.415

1612.403

1611.669

1610.550

1608.992

1611.701

1611.403

1610.797

1609.678

1608.121

1606.137

1610.681

1609.922

1608.802

1607.245

1605.262

1602.866

1609.946

1609.622

1609.037

1607.921

1606.364

1604.381

1601.985

1599.193

1608.938

1608.155

1607.034

1605.478

1603.495

1601.100

1598.309

1595.141

1608.170

1607.819

1607.255

1606.142

1604.586

1602.605

1600.210

1597.419

1594.253

1607.174

1606.367
NOMBRE D IONDES OBSERVE

1615.157
1614.911
1614.103
1613.436
1613.436
1613.174
1612.554
1611.415
1612.394
1611.685
1610.592
1609.038
1611.685

1611.415

1610.802

1609.635

1608.157

1606.140

1610.680

1609.930

1608.795

1607.248

1605.244

1602.854

1609.930

1609.635

1609.038

1607.923

1606.369

1604.350

1601.972

1599.226

1608.937

1608.157

1607.033

1605.467

1603.522

1601.177

1598.295

1595.205

$1608 \cdot 157$

1607.824

$1607 \cdot 248$

1606.140

1604.573

1602.625

1600.224

1597.420

1594.255

1607.176

1606.369
.006

.010

.000

.045

.000

.011

.020

.000

$-.009$

.016

.042

.046

$-.016$

.012

.005

$-.043$

.036

.003

$-.001$

.008

$-.007$

.003

$-.018$

$-.012$

$-.016$

.013

.001

.002

.005

$-.031$

$-.013$

.033

$-.001$

.002

$-.001$

$-.011$

.027

.077

$-.014$

.064

$-.013$

.005

$-.007$

$-.002$

$-.013$

.020

.014

.001

.002

.002

.002
ENERGIE

DU NIVEAU

DE BASE

2.532

10.145

12.572

35.344

8.440

16.135

38.722

76.451

20.065

42.942

80.673

133.276

17.722

25.548

48.011

85.741

138.345

205.607

30.887

53.917

91.652

144.258

211.522

293.179

30.376

38.382

60.681

98.408

151.016

218.283

299.944

395.686

45.036

68.267

106.009

158.619

225.889

307.553

403.300

512.783

46.398

54.637

76.734

114.454

167.066

234.339

316.008

411.7 .60

521.248

62.513

85.991

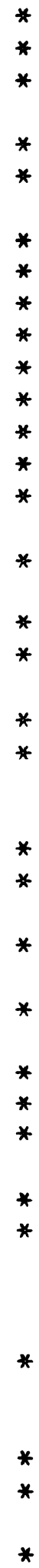


TABLEAU III $b$.

NOMBRES QUANTIQUES

\begin{tabular}{|c|c|c|c|c|c|}
\hline$N^{\prime \prime}$ & $\mathrm{K}:$ & $\mathrm{K}_{+1}^{\prime \prime}$ & $\mathbf{N}^{\prime}$ & & \\
\hline 11 & 3 & 9 & 10 & 3 & \\
\hline 11 & 4 & 8 & 10 & 4 & \\
\hline 11 & 5 & 7 & 10 & 5 & \\
\hline 11 & 6 & 6 & 10 & 6 & \\
\hline 11 & 7 & 5 & 10 & & \\
\hline 11 & 8 & 4 & 10 & 8 & \\
\hline 12 & 0 & 12 & 11 & 0 & \\
\hline 12 & 1 & 11 & 11 & 1 & \\
\hline 12 & 2 & 10 & 11 & 2 & \\
\hline 12 & 3 & 9 & 11 & 3 & \\
\hline 12 & 4 & 8 & 11 & 4 & \\
\hline 12 & 5 & 7 & 11 & 5 & \\
\hline 12 & 6 & 6 & 11 & 6 & \\
\hline 12 & 7 & 5 & 11 & 7 & \\
\hline 12 & 8 & 4 & 11 & 8 & \\
\hline 13 & 1 & 13 & 12 & 1 & \\
\hline 13 & 2 & 12 & 12 & 2 & \\
\hline 13 & 3 & 11 & 12 & 3 & \\
\hline 13 & 4 & 10 & 12 & 4 & \\
\hline 13 & 5 & 9 & 12 & 5 & \\
\hline 13 & 6 & 8 & 12 & 6 & \\
\hline 13 & 7 & 7 & 12 & 7 & \\
\hline 13 & 8 & 6 & 12 & 8 & \\
\hline 14 & 0 & 14 & 13 & 0 & \\
\hline 14 & 1 & 13 & 13 & 1 & \\
\hline 14 & 2 & 12 & 13 & 2 & \\
\hline 14 & 3 & 11 & 13 & 3 & \\
\hline 14 & 4 & 10 & 13 & 4 & \\
\hline 14 & 5 & 9 & 13 & 5 & \\
\hline 14 & 6 & 8 & 13 & 6 & \\
\hline 14 & 7 & 7 & 13 & 7 & \\
\hline 14 & 8 & 6 & 13 & 8 & \\
\hline 15 & 1 & 15 & 14 & 1 & \\
\hline 15 & 2 & 14 & 14 & 2 & \\
\hline 15 & 3 & 13 & 14 & 3 & \\
\hline 15 & 4 & 12 & 14 & 4 & \\
\hline 15 & 5 & 11 & 14 & 5 & \\
\hline 15 & 6 & 10 & 14 & 6 & \\
\hline 15 & 7 & 9 & 14 & 7 & \\
\hline 15 & 8 & 8 & 14 & 8 & \\
\hline 16 & 0 & 16 & 15 & 0 & \\
\hline 16 & 1 & 15 & 15 & 1 & \\
\hline 16 & 2 & 14 & 15 & 2 & \\
\hline 16 & 3 & 13 & 15 & 3 & \\
\hline 16 & 4 & 12 & 15 & 4 & \\
\hline 16 & 5 & 11 & 15 & 5 & \\
\hline 16 & 6 & 10 & 15 & 6 & \\
\hline 16 & 7 & 9 & 15 & 7 & \\
\hline 16 & 8 & 8 & 15 & 8 & \\
\hline 17 & 1 & 17 & 16 & & \\
\hline & 2 & 16 & 16 & & \\
\hline
\end{tabular}

\section{NOMBRE}

D.ONDES

CALCULE

1605.245

1603.690

1601.709

1599.315

1596.526

1593.360

1606.376

1605.996

1605.450

$1604 \cdot 343$

1602.788

1600.808

1598.415

1595.627

1592.463

1605.390

1604.557

1603.435

1604.604

1599.902

1597.511

1594.724

1591.560

1604.563

1604.152

1603.622

1602.522

1600.970

1598.992

1596.601

1593.816

1590.654

1603.585

1602.729

1601.604

1600.052

1598.075

1595.686

1592.902

1589.743

1602.731

1602.287

1601.772

1600.679

1599.130

1597.155

1594.767

1591.985

1588.828

1601.760

1600.878

\section{NOMBRE}

DIONDES

OBSERVE

$1605 \cdot 244$
$1603 \cdot 693$
$1601 \cdot 756$
$1599 \cdot 324$
$1596 \cdot 578$
$1593 \cdot 283$
$1606 \cdot 369$
$1605 \cdot 995$
$1605 \cdot 467$
$1604 \cdot 350$
$1602 \cdot 789$
$1600 \cdot 820$

1598.425

1595.624

1592.499

1605.385

1604.573

1603.435

$1604 \cdot 573$

1599.900

1597.520

1594.764

1591.540

$1604 \cdot 573$

1604.154

1603.609

1602.519

1600.964

1599.003

1596.578

1593.844

1590.682

1603.609

1602.738

1601.604

1600.041

1598.064

1595.685

1592.923

1589.792

1602.738

$1602 \cdot 283$

1601.756

160.0 .674

1599.126

1597.131

1594.764

1591.998

1588.872

1601.756

1600.867
$-.001$

.003

.047

.009

.052

$-.077$

$-.007$

$-.001$

.017

.007

.001

- 012

.010

$-.003$

. 036

$-.005$

-016

.000

$-.031$

$-.002$

- 009

- 040

-.020

- 010

-002

$-.013$

$-.003$

-.006

.011

-.023

- 028

- 028

- 024

- 009

- 000

$-.011$

$-.011$

-.001

- 021

.049

- 007

$-.004$

.016

$-.005$

$-.004$

$-.024$

-.003

- 013

- 044

$-.004$

.011
ENERGIE

DU NIVEAU

DE BASE

123.743

176.358

243.635

325.308

421.065

530.560

65.783

74.310

96.172

133.877

186.494

253.775

335.453

$431 \cdot 216$

540.718

83.314

107.089

144.855

194.752

264.760

346.443

442.213

551.723

88.525

97.399

118.999

156.679

209.300

276.589

358.278

454.055

563.573

107.439

131.557

169.345

221.970

289.264

370.959

466.743

576.269

114.619

123.902

145.218

182.859

235.484

302.782

384.484

480.276

589.811

134.885

159.396

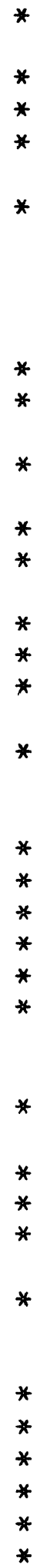


TABLEAU III $c$.

NOMBRES QUANTIQUES

N" $K_{-1}^{n} K_{+1}^{\prime \prime} N^{\prime} K_{-1}^{\prime} K_{+1}^{\prime}$

$\begin{array}{llllll}17 & 3 & 15 & 16 & 3 & 14\end{array}$

$\begin{array}{llllll}17 & 4 & 14 & 16 & 4 & 13\end{array}$

$\begin{array}{llllll}17 & 5 & 13 & 16 & 5 & 12\end{array}$

$\begin{array}{llllll}17 & 6 & 12 & 16 & 6 & 11\end{array}$

$\begin{array}{llllll}17 & 7 & 11 & 16 & 7 & 10\end{array}$

$\begin{array}{llllll}17 & 8 & 10 & 16 & 8 & 9\end{array}$

$\begin{array}{llllll}18 & 0 & 18 & 17 & 0 & 17\end{array}$

$\begin{array}{llllll}18 & 1 & 17 & 17 & 1 & 16\end{array}$

$\begin{array}{llllll}18 & 2 & 16 & 17 & 2 & 15\end{array}$

$\begin{array}{llllll}18 & 3 & 15 & 17 & 3 & 14\end{array}$

$\begin{array}{llllll}18 & 4 & 14 & 17 & 4 & 13\end{array}$

$\begin{array}{llllll}18 & 5 & 13 & 17 & 5 & 12\end{array}$

$\begin{array}{llllll}18 & 6 & 12 & 17 & 6 & 11\end{array}$

$\begin{array}{llllll}18 & 7 & 11 & 17 & 7 & 10\end{array}$

$\begin{array}{llllll}19 & 1 & 19 & 18 & 1 & 18\end{array}$

$\begin{array}{llllll}19 & 2 & 18 & 18 & 2 & 17\end{array}$

$\begin{array}{llllll}19 & 3 & 17 & 18 & 3 & 16\end{array}$

$\begin{array}{llllll}19 & 4 & 16 & 18 & 4 & 15\end{array}$

$\begin{array}{llllll}19 & 5 & 15 & 18 & 5 & 14\end{array}$

$\begin{array}{llllll}19 & 6 & 14 & 18 & 6 & 13\end{array}$

$\begin{array}{llllll}19 & 7 & 13 & 18 & 7 & 12\end{array}$

$\begin{array}{llllll}20 & 0 & 20 & 19 & 0 & 19\end{array}$

$\begin{array}{llllll}20 & 1 & 19 & 19 & 1 & 18\end{array}$

$\begin{array}{llllll}20 & 2 & 18 & 19 & 2 & 17\end{array}$

$\begin{array}{llllll}20 & 3 & 17 & 19 & 3 & 16\end{array}$

$\begin{array}{llllll}20 & 4 & 16 & 19 & 4 & 15\end{array}$

$\begin{array}{llllll}20 & 5 & 15 & 19 & 5 & 14\end{array}$

$\begin{array}{llllll}20 & 6 & 14 & 19 & 6 & 13\end{array}$

$\begin{array}{lll}20 & 7 & 13\end{array}$

$\begin{array}{lll}21 & 1 & 21\end{array}$

21220

$\begin{array}{lll}21 & 3 & 19\end{array}$

$\begin{array}{lll}21 & 4 & 18\end{array}$

$\begin{array}{lll}21 & 5 & 17\end{array}$

$\begin{array}{lll}21 & 6 & 16\end{array}$

$\begin{array}{lll}22 & 0 & 22\end{array}$

$22 \quad 121$

$22 \quad 220$

$\begin{array}{lll}22 & 3 & 19\end{array}$

$\begin{array}{lll}22 & 4 & 18\end{array}$

$\begin{array}{lll}22 & 5 & 17\end{array}$

22616

23123

$\begin{array}{lll}23 & 2 & 22\end{array}$

$\begin{array}{lll}23 & 3 & 21\end{array}$

$\begin{array}{lll}23 & 4 & 20\end{array}$

$\begin{array}{lll}23 & 5 & 19\end{array}$

240024

24123

$\begin{array}{lll}24 & 222\end{array}$

24321
NOMBRE

DIONDES

CALCULE

1599.752

1598.203

1596.229

1593.842

1591.062

1587.907

1600.881

1600.402

1599.898

1598.817

1597.270

1595.297

1592.913

1590.134

1599.916

1599.008

1597.878

1596.332

1594.361

1591.979

1589.203

1599.013

1598.496

1598.001

1596.932

1595.390

1593.421

1591.040

1588.266

1598.050

1597.116

1595.984

1594.441

1592.474

1590.096

1597.126

1596.571

1596.081

1595.027

1593.488

1591.523

1589.147

1596.166

1595.206

1594.069

1592.530

1590.566

1595.221

1594.624

1594.136

1593.099
NOMBRE

DIONDES OBSERVE

ENERGIE

OBS-CALC DU NIVEAU DE BASE

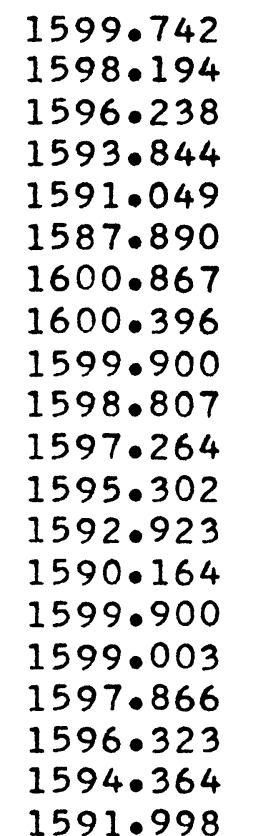

197.212

$-.00$

.009

249.842

.002

$-.013$

$-.017$

$-.014$

$-.006$

.002

$-.010$

$-.006$

.005

.010

.030

$-.016$

$-.005$

$-.012$

$-.009$

.003

.019

1589.213

1599.003

1598.486

1598.006

1596.923

1595.377

1593.444

1591.049

1588.272

1598.064

1597.131

1595.974

1594.431

1592.499

1590.086

1597.131

1596.578

1596.075

1595.040

1593.444

1591.540

1589.159

1596.159

1595.205

1594.052

1592.499

1590.580

1595.205

1594.619

$1594 \cdot 129$

1593.091
.010

$-.010$

$-.010$

.005

$-.009$

$-.013$

.023

.009

.006

.014

.015

$-.010$

$-.010$

.025

$-.010$

.005

.007

$-.006$

.013

. .044

.017

.012

$-.007$

$-.001$

$-.017$

$-.031$

.014

$-.016$

$-.005$

$-.007$

$-.008$
317.145

398.854

494.654

604.199

144.057

153.816

174.834

212.416

265.044

332.353

414.068

509.878

165.649

190.601

228.456

281.091

348.405

430.127

525.946

176.832

187.139

207.851

245.352

297.981

365.300

447.031

542.859

199.730

225.172

263.077

315.716

383.040

464.779

212.935

223.865

244.273

281.666

334.294

401.624

483.371

237.123

263.104

301.073

353.716

421.052

252.358

263.992

284.107

321.360 
TABLEAU III $d$.

NOMBRES QUANTI QUES

$N^{\prime \prime} \quad K_{-1}^{\prime \prime} K_{+1}^{\prime \prime} N^{\prime} \quad K_{-1}^{\prime} K_{+1}^{\prime}$

$\begin{array}{llllll}24 & 4 & 20 & 23 & 4 & 19\end{array}$

$\begin{array}{llllll}24 & 5 & 19 & 23 & 5 & 18\end{array}$

$\begin{array}{llllll}25 & 1 & 25 & 24 & 1 & 24\end{array}$

$\begin{array}{llllll}25 & 2 & 24 & 24 & 2 & 23\end{array}$

$\begin{array}{llllll}25 & 3 & 23 & 24 & 3 & 22\end{array}$

$\begin{array}{llllll}25 & 4 & 22 & 24 & 4 & 21\end{array}$

$\begin{array}{llllll}25 & 5 & 21 & 24 & 5 & 20\end{array}$

$\begin{array}{llllll}26 & 0 & 26 & 25 & 0 & 25\end{array}$

$\begin{array}{llllll}26 & 1 & 25 & 25 & 1 & 24\end{array}$

$\begin{array}{llllll}26 & 2 & 24 & 25 & 2 & 23\end{array}$

$\begin{array}{llllll}26 & 3 & 23 & 25 & 3 & 22\end{array}$

$26 \quad 4 \quad 22 \quad 25 \quad 4 \quad 21$

$\begin{array}{llllll}26 & 5 & 21 & 25 & 5 & 20\end{array}$

27127

27226

$\begin{array}{lll}27 & 3 & 25\end{array}$

$27 \quad 424$

280028

$\begin{array}{lll}28 & 1 & 27\end{array}$

$\begin{array}{lll}28 & 2 & 26\end{array}$

$\begin{array}{lll}28 & 3 & 25\end{array}$

28424

29129

$\begin{array}{lll}29 & 2 & 28\end{array}$

$\begin{array}{lll}29 & 3 & 27\end{array}$

30030

30129

30228

000

$\begin{array}{lll}1 & 1 & 1\end{array}$

202

$\begin{array}{lll}2 & 1 & 1\end{array}$

220

313

322

331

404

413

422

431

440

515

524

533

542

$\begin{array}{lll}5 & 5 & 1\end{array}$

606

615

624

633

642

26126

101
NOMBRE

DIONDES

CALCULE

1591.566

1589.606

1594.260

1593.274

1592.133

1590.598

1588.639

1593.299

1592.659

1592.169

1591.151

1589.624

1587.668

26225

$\begin{array}{lll}26 & 3 & 24\end{array}$

26

27

27

27

27
27

28

28

28

29029

29

$\begin{array}{lll}29 & 2 & 27\end{array}$

$\begin{array}{lll}2 & 1 & 2\end{array}$

303

312

321

414

423

432

505

514

523

532

541

616

625

634

643

$\begin{array}{lll}6 & 4 & 3 \\ 6 & 5 & 2\end{array}$

$\begin{array}{lll}7 & 0 & 7\end{array}$

$\begin{array}{lll}7 & 1 & 6\end{array}$

725

$\begin{array}{lll}7 & 3\end{array}$
1592.335

1591.322

1590.176

1588.645

1591.359

1590.672

1590.179

1589.181

1587.661

1590.390

1589.351

1588.198

1589.400

1588.668

1588.166

1617.683

1618.265

1619.344

1619.153

1618.445

1619.896

1619.267

1618.150

1620.983

1620.816

1620.086

1618.968

1617.414

1621.503

1620.897

1619.781

1618.227

1622.600

1622.456

1621.707

1620.588

1619.035
1616.248
NOMBRE

D.ONDES

OBSERVE

OBS-CALC DU NIVEAU

DE NIVEAU

$1591 \cdot 540$

1589.612

$1594 \cdot 255$

1593.283

$1592 \cdot 146$

1590.580

1588.649

1593.283

1592.661

1592.146

$1591 \cdot 133$

1589.612

1587.662

$1592 \cdot 334$

$1591 \cdot 338$

$1590 \cdot 164$

1588.640

1591.338

1590.682

1590.164

1589.213

1587.662

1590.383

1589.375

1588.178

1589.375

1588.649

$1588 \cdot 178$

1617.685

1618.251

1619.336

1619.15

1618.411

1619.889

1619.262

1618.113

1620.998

1620.814

1620.093

1618.942

1617.421

1621.499

1620.902

1619.785

1618.251

1616.225

1622.595

1622.491

1621.717

1620.618

1619.016
$-.026$

.006

$-.005$

.009

.013

-.018

- 010

..016

.002

$-.023$

$-.018$

$-.012$

$-.006$

$-.001$

.016

$-.012$

$-.005$

$-.021$

.010

$-.015$

.032

.001

$-.007$

.024

$-.020$

$-.025$

$-.019$

.012

.002

$-.014$

$-.008$

.006

$-.034$

$-.007$

$-.005$

-.037

.015

$-.002$

.007

. .026

.007

$-.004$

.005

.004

- 024

.023

$-.005$

.035

- 010

.030

$-.019$
373.983

441.323

277.827

$304 \cdot 396$

342.445

395.092

462.438

295.091

307.513

327.354

364.432

417.045

484.396

321.838

349.045

387.191

439.841

341.126

$354 \cdot 426$

374.020

410.884

463.481

369.152

397.046

435.310

390.455

404.722

$424 \cdot 108$ .000

8.410

2.532

10.145

32.811

12.572 
TABLEAU III $e$.

NOMBRES QUANTI QUES

\begin{tabular}{|c|c|c|c|c|c|c|}
\hline $\mathrm{N}^{\prime \prime}$ & $K_{-1}^{\prime \prime}$ & $K_{+1}^{\prime \prime}$ & $N^{\prime}$ & $K_{-1}^{\prime}$ & $K_{+1}^{\prime \prime}$ & CALCULE \\
\hline 6 & 5 & 1 & 7 & 5 & 2 & 1617.057 \\
\hline 6 & 6 & 0 & 7 & 6 & 1 & 1614.668 \\
\hline 7 & 1 & 7 & 8 & 1 & 8 & 1623.087 \\
\hline 7 & 2 & 6 & 8 & 2 & 7 & 1622.505 \\
\hline 7 & 3 & 5 & 8 & 3 & 6 & 1621.391 \\
\hline 7 & 4 & 4 & 8 & 4 & 5 & 1619.839 \\
\hline 7 & 5 & 3 & 8 & 5 & 4 & 1617.862 \\
\hline 7 & 6 & 2 & 8 & 6 & 3 & 1615.474 \\
\hline 7 & 7 & 1 & 8 & 7 & 2 & 1612.691 \\
\hline 8 & 0 & 8 & 9 & 0 & 9 & 1624.192 \\
\hline 8 & 1 & 7 & 9 & 1 & 8 & 1624.074 \\
\hline 8 & 2 & 6 & 9 & 2 & 7 & $1623 \cdot 308$ \\
\hline 8 & 3 & 5 & 9 & 3 & 6 & 1622.188 \\
\hline 8 & 4 & 4 & 9 & 4 & 5 & 1620.636 \\
\hline 8 & 5 & 3 & 9 & 5 & 4 & 1618.661 \\
\hline 8 & 6 & 2 & 9 & 6 & 3 & $1616 \cdot 274$ \\
\hline 8 & 7 & 1 & 9 & 7 & 2 & 1613.493 \\
\hline 8 & 8 & 0 & 9 & 8 & 1 & 1610.338 \\
\hline 9 & 1 & 9 & 10 & 1 & 10 & 1624.651 \\
\hline 9 & 2 & 8 & 10 & 2 & 9 & 1624.091 \\
\hline 9 & 3 & 7 & 10 & 3 & 8 & 1622.979 \\
\hline 9 & 4 & 6 & 10 & 4 & 7 & 1621.429 \\
\hline 9 & 5 & 5 & 10 & 5 & 6 & 1619.455 \\
\hline 9 & 6 & 4 & 10 & 6 & 5 & 1617.070 \\
\hline 9 & 7 & 3 & 10 & 7 & 4 & $1614 \cdot 291$ \\
\hline 9 & 8 & 2 & 10 & 8 & 3 & 1611.137 \\
\hline 10 & 0 & 10 & 11 & 0 & 11 & 1625.761 \\
\hline 10 & 1 & 9 & 11 & 1 & 10 & 1625.669 \\
\hline 10 & 2 & 8 & 11 & 2 & 9 & $1624 \cdot 888$ \\
\hline 10 & 3 & 7 & 11 & 3 & 8 & 1623.766 \\
\hline 10 & 4 & 6 & 11 & 4 & 7 & 1622.216 \\
\hline 10 & 5 & 5 & 11 & 5 & 6 & 1620.244 \\
\hline 10 & 6 & 4 & 11 & 6 & 5 & 1617.860 \\
\hline 10 & 7 & 3 & 11 & 7 & 4 & 1615.083 \\
\hline 10 & 8 & 2 & 11 & 8 & 3 & 1611.933 \\
\hline 11 & 1 & 11 & 12 & 1 & 12 & 1626.191 \\
\hline 11 & 2 & 10 & 12 & 2 & 11 & 1625.655 \\
\hline 11 & 3 & 9 & 12 & 3 & 10 & 1624.547 \\
\hline 11 & 4 & 8 & 12. & 4 & 9 & 1622.998 \\
\hline 11 & 5 & 7 & 12 & 5 & 8 & 1621.027 \\
\hline 11 & 6 & 6 & 12 & 6 & 7 & 1618.646 \\
\hline 11 & 7 & 5 & 12 & 7 & 6 & 1615.872 \\
\hline 11 & 8 & 4 & 12 & 8 & 5 & 1612.723 \\
\hline 12 & 0 & 12 & 13 & 0 & 13. & 1627.305 \\
\hline 12 & 1 & 11 & 13 & 1 & 12 & 1627.241 \\
\hline 12 & 2 & 10 & 13 & 2 & 11 & 1626.449 \\
\hline 12 & 3 & 9 & 13 & 3 & 10 & $1625 \cdot 324$ \\
\hline 12 & 4 & 8 & 13 & 4 & 9 & 1623.776 \\
\hline 12 & 5 & 7 & 13 & 5 & 8 & 1621.806 \\
\hline 12 & 6 & 6 & 13 & 6 & 7 & 1619.426 \\
\hline 12 & 7 & 5 & 13 & 7 & 6 & 1616.655 \\
\hline
\end{tabular}

NOMBRE D.ONDES OBSERVE

\subsection{7}

1614.656

1623.093

$1622 \cdot 491$

$1621 \cdot 408$

1619.889

1617.836

1615.443

1612.722

1624.197

1624.093

1623.312

1622.202

1620.618

1618.641

1616.225

1613.436

1610.324

1624.655

1624.093

1622.991

1621.408

1619.437

1617.037

1614.284

1611.217

1625.764

1625.649

1624.890

1623.779

1622.202

1620.236

1617.836

1615.066

1611.942

1626.201

1625.649

1624.551

1622.991

1620.998

1618.641

1615.911

1612.722

1627.303

1627.222

1626.451

1625.321

1623.779

1621.822

1619.437

1616.643
ENERGIE

OBS-CALC DU NIVEAU DE BASE

$\begin{array}{rr}-.020 & 2 \\ -.012 & 2 \\ .006 & \\ -.014 & \\ .017 & \\ .050 & 1 \\ -.026 & 2 \\ -.031 & 2 \\ .031 & 3\end{array}$

$205.607 \quad *$

287.261 *

30.887

53.917

91.652

$144 \cdot 258$

$211 \cdot 522$

293.179

388.918

30.376

38.382

60.681

98.408

151.016

218.283

299.944

395.686

505.163

45.036

68.267

106.009

158.619

225.889

307.553

403.300

512.783

46.398

$54 \cdot 637$

76.734

114.454

167.066

234.339

316.008

411.760

521.248

62.513

85.991

123.743

.004

176.358

243.635

325.308

421.065

530.560

65.783

74.310

96.172

133.877

186.494

253.775

335.453

431.216 
REVUE DE PHYSIQUE APPLIQUÉE

TABLeAU III $f$.

NOMBRES QUANTIQUES

$N^{\prime \prime} \quad K_{-1}^{\prime \prime} K_{+1}^{\prime \prime} N^{\prime} K_{-1}^{\prime} K_{+1}^{\prime}$

$\begin{array}{llllll}12 & 8 & 4 & 13 & 8 & 5\end{array}$

$\begin{array}{llllll}13 & 1 & 13 & 14 & 1 & 14\end{array}$

$\begin{array}{llllll}13 & 2 & 12 & 14 & 2 & 13\end{array}$

$\begin{array}{llllll}13 & 3 & 11 & 14 & 3 & 12\end{array}$

$\begin{array}{llllll}13 & 4 & 10 & 14 & 4 & 11\end{array}$

$\begin{array}{llllll}13 & 5 & 9 & 14 & 5 & 10\end{array}$

$\begin{array}{llllll}13 & 6 & 8 & 14 & 6 & 9\end{array}$

$\begin{array}{llllll}13 & 7 & 7 & 14 & 7 & 8\end{array}$

138614

$140014 \quad 15$

14113

14212

14311

14410

1459

146

147

148

15115

$\begin{array}{lll}15 & 2 & 14\end{array}$

$\begin{array}{lll}15 & 3 & 13\end{array}$

15412

$15 \quad 511$

15610

$\begin{array}{lll}15 & 7\end{array}$

1588

$16 \quad 0 \quad 16$

$\begin{array}{lll}16 & 1 & 15\end{array}$

$\begin{array}{lll}16 & 2 & 14\end{array}$

$\begin{array}{lll}16 & 3 & 13\end{array}$

$164 \quad 12$

$\begin{array}{lll}16 & 5 & 11\end{array}$

$16 \quad 6 \quad 10$

$\begin{array}{lll}16 & 7\end{array}$

16

17

17

17

17

17

17

17

17

18

$\begin{array}{lllll}18 & 17 & 19 & 19\end{array}$

$18216 \quad 19 \quad 118$

$\begin{array}{llllll}18 & 3 & 15 & 19 & 3 & 16\end{array}$

$\begin{array}{llllll}18 & 4 & 14 & 19 & 4 & 15\end{array}$

$\begin{array}{llllll}18 & 5 & 13 & 19 & 5 & 14\end{array}$

$\begin{array}{llllll}18 & 6 & 12 & 19 & 6 & 13\end{array}$

$\begin{array}{llllll}18 & 7 & 11 & 19 & 7 & 12\end{array}$
NOMBRE

D'ONDES CALCULE

\subsection{9}

1627.710

1627.197

1626.094

1627.270

1622.579

1620.202

1617.432

1614.289

1628.825

1628.790

1627.991

1626.859

1625.314

1623.348

1620.973

1618.206

1615.066

1629.206

1628.717

1627.619

1626.075

1624.110

1621.737

1618.973

1615.837

1630.319

1630.316

1629.514

1628.374

1626.830

1624.868

1622.497

1619.736

1616.604

1630.680

1630.213

1629.122

1627.581

1625.621

1623.252

1620.495

1617.366

1631.788

1631.819

1631.018

1629.868

1628.327

1626.368

1624.003

1621.247
NOMBRE D'ONDES OBSERVE OBS-CALC DU NIVEAU DE BASE

$1613 \cdot 436$

$-.073$

1627.708

1627.222

1626.089

1627.303

1622.595

1620.236

1617.421

$1614 \cdot 284$

1628.807

1628.807

1627.991

1626.842

1625.321

1623.312

1620.998

$1618 \cdot 251$

1615.066

1629.202

1628.717

1627.608

1626.089

1624.093

1621.717

1618.942

1615.911

1630.323

1630.323

1629.510

$1628 \cdot 348$

1626.842

1624.890

1622.491

1619.785

1616.643

1630.685

1630.218

1629.087

1627.608

1625.649

1623.312

1620.618

1617.421

1631.824

1631.824

1631.020

1629.857

1628.348

1626.371

1623.995

$1621 \cdot 263$
$-.002$

.025

$-.005$

.033

.016

.034

$-.011$

$-.005$

$-.018$

.017

.000

$-.017$

.007

$-.036$

.025

.045

.000

$-.004$

.000

$-.011$

.014

$-.017$

$-.020$

$-.031$

.074

.004

.007

$-.004$

$-.026$

.012

.022

$-.006$

.049

.039

.005

.005

$-.035$

.027

.028

.060

.123

.055

.036

.005

.002

$-.011$

.021

.003

$-.008$

.016
540.718

83.314

107.089

144.855

194.752

264.760

346.443

442.213

551.723

88.525

97.399

118.999

156.679

209.300

276.589

358.278

454.055

563.573

107.439

131.557

169.345

221.970

289.264

370.959

466.743

576.269

114.619

123.902

145.218

182.859

235.484

302.782

384.484

480.276

589.811

134.885

159.396

197.212

249.842

317.145

398.854

494.654

604.199

144.057

153.816

174.834

212.416

265.044

332.353

414.068

509.878 
TABleAU III $g$.

\section{NOMBRES QUANTIQUES}

$N^{\prime \prime} K_{-1}^{\prime \prime} K_{+1}^{\prime \prime} N^{\prime} K_{-1}^{\prime} K_{+1}^{\prime}$

18

119

$$
19
$$

811

20120

$\begin{array}{llllll}19 & 2 & 18 & 20 & 2 & 19\end{array}$

$19 \quad 3 \quad 17 \quad 20 \quad 318$

$\begin{array}{llllll}19 & 4 & 16 & 20 & 4 & 17\end{array}$

19515

19614

19713

$\begin{array}{lll}19 & 8 & 12\end{array}$

$20 \quad 0 \quad 20$

$20 \quad 119$

$20 \quad 2 \quad 18$

$20 \quad 317$

$20 \quad 4 \quad 16$

$20 \quad 5 \quad 15$

$\begin{array}{lll}20 & 6 & 14\end{array}$

20713

$20 \quad 8 \quad 12$

$\begin{array}{lll}21 & 1 & 21\end{array}$

21220

21319

$\begin{array}{lll}21 & 4 & 18\end{array}$

$\begin{array}{lll}21 & 5 & 17\end{array}$

21616

21715

$21 \quad 8 \quad 14$

$\begin{array}{lll}22 & 0 & 22\end{array}$

$\begin{array}{lll}22 & 1 & 21\end{array}$

22220

$\begin{array}{lll}22 & 3 & 19\end{array}$

$\begin{array}{lll}22 & 4 & 18\end{array}$

$\begin{array}{lll}22 & 5 & 17\end{array}$

22616

22715

$\begin{array}{lll}22 & 8 & 14\end{array}$

23123

$\begin{array}{lll}23 & 2 & 22\end{array}$

$23 \quad 3 \quad 21$

23420

23519

$\begin{array}{lll}23 & 6 & 18\end{array}$

$\begin{array}{lll}23 & 7 & 17\end{array}$

23816

24024

24123

$\begin{array}{lll}24 & 22\end{array}$

24321

24420

24519

$\begin{array}{lll}24 & 6 & 18\end{array}$

24717
NOMBRE

D'ONDES

CALCULE

1618.122

1632.131

1631.687

1630.605

1629.066

1627.109

1624.748

1621.996

1618.875

1633.229

1633.297

1632.503

1631.341

1629.801

1627.847

1625.487

1622.739

1619.622

1633.559

1633.138

1632.065

1630.530

1628.578

1626.221

1623.477

1620.364

1634.644

1634.751

1633.970

1632.793

1631.255

1629.305

1626.951

1624.211

1621.101

1634.964

1634.566

1633.505

1631.974

1630.025

1627.675

1624.939

1621.834

1636.032

1636.180

1635.416

1634.223

1632.686

1630.741

1628.394

1625.661
NOMBRE DיONDES OBSERVE

ENERGIE

1618.113

1632.153

1631.713

1630.602

1629.087

1627.118

1624.744

1621.999

1618.870

1633.227

1633.303

1632.521

1631.337

1629.792

1627.845

1625.473

1622.737

1619.633

1633.569

1633.141

1632.065

1630.510

1628.592

1626.201

1623.473

1620.403

1634.652

1634.764

1633.972

1632.790

$1631 \cdot 244$

$1629 \cdot 316$

1626.933

1624.197

1620.998

1634.945

1634.565

1633.501

1631.964

1630.044

1627.608

1624.890

1621.822

1636.039

1636.165

1635.422

1634.237

1632.673

1630.764

$1628 \cdot 348$

1625.649
-.009
.022
.026
-.003
.021
.009
-.004
.003

$-.005$

$-.002$

.006

.018

$-.004$

$-.009$

$-.002$

.014

$-.002$

.011

.010

.003

$-.000$

$-.020$

.014

$-.020$

$-.004$

.039

.008

.013

.002

$-.003$

$-.011$

.011

$-.018$

$-.014$

$-.103$

$-.019$

$-.001$

$-.004$

$-.010$

.019

$-.067$

$-.049$

$-.012$

.007

$-.015$

.006

.014

. .013

.023

. .046

$-.012$
U NIVEAU

DE BASE

619.433

165.649

190.601

228.456

281.091

348.405

430.127

525.946

635.512

176.832

187.139

207.851

245.352

297.981

365.300

447.031

542.859

652.437

199.730

225.172

263.077

315.716

383.040

464.779

560.617

670.207

212.935

223.865

$244 \cdot 273$

281.666

334.294

401.624

483.371

579.219

688.822

237.123

263.104

353.716

421.052

502.807

598.666

708.282

252.358

263.992

284. 107

321.360

373.983

441.323

523.087

618.958
301.073

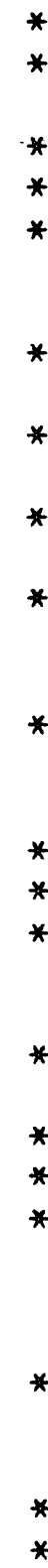


TABLEAU III $h$.

NOMBRES QUANTIQUES

$N^{\prime \prime} \quad K_{-1}^{\prime \prime} K_{+1}^{\prime \prime} \quad N^{\prime} K_{-1}^{\prime} K_{+1}^{\prime}$

\begin{tabular}{|c|c|c|c|c|c|}
\hline 24 & 16 & 25 & 8 & 17 & 1622.563 \\
\hline 25 & 125 & 26 & 1 & 26 & 1636.346 \\
\hline 25 & 24 & 26 & 2 & 25 & 1635.971 \\
\hline 25 & 23 & 26 & 3 & 24 & $1634 \cdot 922$ \\
\hline 25 & 422 & 26 & 4 & 23 & 1633.394 \\
\hline 25 & 521 & 26 & 5 & 22 & 1631.452 \\
\hline 25 & 620 & 26 & 6 & 21 & 1629.108 \\
\hline 25 & 719 & 26 & 7 & 20 & 1626.380 \\
\hline 25 & 818 & 26 & 8 & 19 & 1623.285 \\
\hline 26 & 026 & 27 & 0 & 27 & 1637.394 \\
\hline 26 & 125 & 27 & 1 & 26 & 1637.585 \\
\hline 26 & 224 & 27 & 2 & 25 & 1636.845 \\
\hline 26 & 323 & 27 & 3 & 24 & 1635.633 \\
\hline 26 & 422 & 27 & 4 & 23 & 1634.097 \\
\hline 26 & 521 & 27 & 5 & 22 & 1632.157 \\
\hline 26 & 620 & 27 & 6 & 21 & 1629.817 \\
\hline 26 & 719 & 27 & 7 & 20 & 1627.092 \\
\hline 26 & 818 & 27 & 8 & 19 & 1624.003 \\
\hline 27 & 127 & 28 & 1 & 28 & 1637.704 \\
\hline 27 & 26 & 28 & 2 & 27 & 1637.352 \\
\hline 27 & 25 & 28 & 3 & 26 & 1636.317 \\
\hline 27 & 24 & 28 & 4 & 25 & 1634.794 \\
\hline 27 & 23 & 28 & 5 & 24 & 1632.857 \\
\hline 27 & 22 & 28 & 6 & 23 & 1630.521 \\
\hline 27 & 21 & 28 & 7 & 22 & 1627.801 \\
\hline 27 & 20 & 28 & 8 & 21 & 1624.727 \\
\hline 28 & 28 & 29 & 0 & 29 & 1638.729 \\
\hline 28 & 27 & 29 & 1 & 28 & 1638.964 \\
\hline 28 & 26 & 29 & 2 & 27 & 1638.254 \\
\hline 28 & 25 & 29 & 3 & 26 & 1637.023 \\
\hline 28 & 24 & 29 & 4 & 25 & 1635.487 \\
\hline 28 & 23 & 29 & 5 & 24 & 1633.551 \\
\hline 28 & 22 & 29 & 6 & 23 & 1631.219 \\
\hline 28 & 21 & 29 & 7 & 22 & $16 ? .8 \cdot 504$ \\
\hline 28 & 20 & 29 & 8 & 21 & 1625.425 \\
\hline 29 & 29 & 30 & 1 & 30 & 1639.040 \\
\hline 29 & 28 & 30 & 2 & 29 & 1638.709 \\
\hline 29 & 27 & 30 & 3 & 28 & 1637.691 \\
\hline 29 & 26 & 30 & 4 & 27 & 1636.173 \\
\hline 29 & 25 & 30 & 5 & 26 & $1634 \cdot 241$ \\
\hline 29 & 24 & 30 & 6 & 25 & 1631.911 \\
\hline 29 & 23 & 30 & 7 & 24 & 1629.201 \\
\hline 29 & 822 & 30 & 8 & 23 & $1626 \cdot 128$ \\
\hline
\end{tabular}

NOMBRE
DIONDES CALCULE

$\begin{array}{ll}1622 \cdot 563 & 1622 \cdot 595 \\ 1636 \cdot 346 & 1636 \cdot 328 \\ 1635 \cdot 971 & 1636 \cdot 001 \\ 1634 \cdot 922 & 1634 \cdot 945 \\ 1633 \cdot 394 & 1633 \cdot 385 \\ 1631.452 & 1631 \cdot 465 \\ 1629 \cdot 108 & 1629 \cdot 087 \\ 1626 \cdot 380 & 1626 \cdot 371 \\ 1623 \cdot 285 & 1623 \cdot 312 \\ 1637 \cdot 394 & 1637 \cdot 380 \\ 1637 \cdot 585 & 1637 \cdot 606 \\ 1636.845 & 1636 \cdot 840 \\ 1635.633 & 1635 \cdot 633 \\ 1634 \cdot 097 & 1634 \cdot 079 \\ 1632 \cdot 157 & 1632 \cdot 153 \\ 1629 \cdot 817 & 1629 \cdot 792 \\ 1627 \cdot 092 & 1627 \cdot 118 \\ 1624 \cdot 003 & 1623 \cdot 995 \\ 1637 \cdot 704 & 1637 \cdot 687 \\ 1637 \cdot 352 & 1637 \cdot 380 \\ 1636 \cdot 317 & 1636 \cdot 328 \\ 1634 \cdot 794 & 1634 \cdot 764 \\ 1632 \cdot 857 & 1632 \cdot 874 \\ 1630 \cdot 521 & 1630 \cdot 510 \\ 1627 \cdot 801 & 1627 \cdot 845 \\ 1624 \cdot 727 & 1624 \cdot 744 \\ 1638 \cdot 729 & 1638 \cdot 732 \\ 1638 \cdot 964 & 1638 \cdot 967 \\ 1638 \cdot 254 & 1638 \cdot 269 \\ 1637 \cdot 023 & 1637 \cdot 013 \\ 1635 \cdot 487 & 1635 \cdot 465 \\ 1633 \cdot 551 & 1633 \cdot 569 \\ 1631 \cdot 219 & 1631 \cdot 244 \\ 1638 \cdot 504 & 1628 \cdot 459 \\ 1625 \cdot 425 & 1625 \cdot 473 \\ 1639 \cdot 040 & 1639 \cdot 042 \\ 1638 \cdot 709 & 1638 \cdot 732 \\ 1637 \cdot 691 & 1637 \cdot 687 \\ 1636 \cdot 173 & 1636 \cdot 165 \\ 1634 \cdot 241 & 1634 \cdot 237 \\ 1631 \cdot 911 & 1631 \cdot 824 \\ 1629 \cdot 201 & 1629 \cdot 202 \\ 1626 \cdot 128 & 1626 \cdot 089\end{array}$

NOMBRE DIONDES OBSERVE ENERGIE
DU NIVEAU DE BASE

$$
\begin{aligned}
& .032 \\
& .018 \\
& \text { - } 030 \\
& \text {.023 } \\
& -.009 \\
& \text {-013 } \\
& -.021 \\
& \text {-.009 } \\
& .027 \\
& .014 \\
& .021 \\
& -.005 \\
& .000 \\
& -.018 \\
& \text {-.004 } \\
& .025 \\
& \text {. } 026 \\
& -.008 \\
& -.017 \\
& \text { - } 028 \\
& \text { - } 011 \\
& \text {-.030 } \\
& .017 \\
& -.011 \\
& .044 \\
& .017 \\
& .003 \\
& .003 \\
& \text { - } 015 \\
& \text {-.010 } \\
& -.022 \\
& \text {-018 } \\
& .025 \\
& .045 \\
& \text {. } 048 \\
& .002 \\
& .023 \\
& -.004 \\
& -.008 \\
& -.004 \\
& -.087 \\
& .001 \\
& -.039
\end{aligned}
$$

28.586

277.827

304.396

342.445

395.092

462.438

544.211

640.093

749.736

295.091

307.513

327.354

364.432

417.045

484.396

566.178

662.073

771.730

321.838

349.045

387.191

439.841

507.198

588.989

684.896

794.568

341.126

354.426

374.020

410.884

463.481

530.843

612.643

708.563

818.251

369.152

397.046

435.310

487.963

555.330

637.141

733.074

842.777

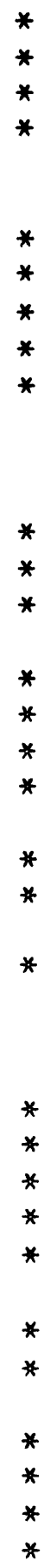

